\title{
Addiction and Evolutionary Process, Common Aspects in Physio-Pathologic Pathways Useful in Pharmaco- Toxicological Approach
}

\author{
Luisetto $\mathbf{M}^{1 *}$, Naseer Almukhtar ${ }^{2}$, Ghulam Rasool Mashori ${ }^{3}$, Behzad Nili Ahmadabadi ${ }^{4}$ and Ram Kumar Sahu ${ }^{5}$ \\ ${ }^{1}$ Applied Pharmacologist, European specialist lab medicine, independent researcher, Italy
}

${ }^{2}$ Professor of physiology, college of medicine, Iraq

${ }^{3}$ Professor of Pharmacology, Pakistan

${ }^{4}$ Nano Drug Delivery (a product development firm), USA

${ }^{5}$ Associate Professor, Pt Deendayal Upadhyay Memorial health science, India

*Corresponding author: Luisetto Mauro, Applied Pharmacologist, European specialist lab medicine, Independent researcher, Italy

\begin{abstract}
Observing vertebrates evolutionary in mammalian are present characteristic pathways involved in progeny take care in first period after birth. In this behavior and instinct, learning neuronal circuits are evolved and reward mechanism the same. In addiction is possible to observe that definite neuronal circuits plays a crucial role. In this work are showed this similarity to better understand the addiction condition. Reward mechanism (primary, intrinsic or extrinsic) are involved in food, reproductive- sexual activity but also in other human condition. Conditioning, reinforce, reward, learning behavior, depression status, level of motivations is common concept involved also in addiction.
\end{abstract}

Keywords: Addiction; Toxicology; Physiology; Anatomy; Mammalians; Vertebrates

\section{Introduction}

Starting from the evolutionary of vertebrates is possible to verify that mammalians vs reptile in example show a great characteristic in cooperative behavior, maternal instinct, high mother depending of progeny and other relevant aspects. So is clear that this kind of vertebrates show a great depending relationship between mother-progeny and we can say that this kind of "animals species" are under a high constraint under an (behavioral, instinct, psychology, neurological aspect). (Anatomically are involved cortex, basal ganglia and thalamus but also other system like amygdala) Dopaminergic- Mesolimbic pathway in example Dopamine is involved in many physiological functions like emotion, pleasure, movement (extrapyramidal), Breast feeding, involved in depression or schizophrenia, prolactin regulation, nausea and vomiting and other. Other molecules involved in "happiness": dopamine, serotonin, endorfin and oxytocin. (oxytocin: role in mother-infant relations, attachment, and bonding in humans). Neuronal circuits of rewards, dopaminergic, glutaminergic, gabaergic (circuits play a relevant role in this mechanism. Breastfeed imply a prolactin and dopaminergic involvement as well as oxytocin and is an evolutionary advantages towards other vertebrates. Viviparous, Parental care of progeny, breast feed, communication abilities between individuals, associative learning, emotional control are common properties of mammalians like emotional, social behavior. (hierarchy, communication to other member of the group of danger and so on).

\section{Material and Methods}

Using an observational approach from biomedical and scientific literature in PUB MED is possible to verify the Neuro- 
pharmacolgical pathways involved in some mammalian behavior and istinct and in addiction condition.

The bibliography reported is chosed related the keywords and related the aim of this work.

\section{Results}

According Biomedical Literature is Possible to Verify that: Archeology science not to be consider only related human product and manufacts but also an inside disciple to verify archeological process related to mind- set kinetics and to other system or organs. Brain, mind, immunologic system and other relevant physiological functions are deeply influenced by a primitive structure and to deeply understand the meaning of this complex system inside us make possible to better explain today Human behavior and physiology and other process [1].

Marco Diana [2]: "Dopamine (DA) transmission is deeply affected by drugs of abuse, and alterations in DA function are involved in the various phases of drug- addiction and potentially exploitable therapeutically. studies have documented a reduction in the electrophysiological activity of DA neurons in alcohol, opiate, cannabinoid, and other drug-dependent rats. DA release in the Nucleus accumbent is decreased in virtually all drug-dependent rodents. these research studies are supported by increments in intracranial self-stimulation (ICSS) thresholds during withdrawal from alcohol, nicotine, opiates, and other drugs of abuse, thereby suggesting a hypofunction of the neural substrate of ICSS. morphological evaluations fed into realistic computational analysis of the medium spiny neuron of the Nacc, post-synaptic counterpart of DA terminals, show profound changes in structure and function of the entire mesolimbic system.

Human imaging studies have shown a reduction of dopamine receptors accompanied by a lesser release of endogenous DA in the ventral striatum of cocaine, heroin, and alcohol-dependent subjects, thereby offering visual proof of the "dopamine-impoverished" addicted human brain. The lasting reduction in physiological activity of the DA system leads to the idea that an increment in its activity, to restore pre-drug levels, may yield significant clinical improvements (reduction of craving, relapse, and drug-seeking/ taking). In theory, it may be achieved pharmacologically and/or with novel interventions such as transcranial magnetic stimulation. Its anatomo-physiological rationale as a possible therapeutic aid in alcoholics and other addicts will be described and proposed as a theoretical framework to be subjected to experimental testing in human addicts [2].

Andrew B Barron et al. [3]: Motile animals actively seek out and gather resources they find rewarding, and this is an extremely powerful organizer and motivator of the animal behavior. Mammalian research studies have revealed interconnected neurobiological systems for reward learning, reward assessment, reinforcement and reward-seeking; all involving the biogenic amine dopamine. The neuro-biology of reward-seeking behavioral systems is less well understood in the invertebrates, but in many diverse invertebrate groups, reward learning and responses to food rewards also involve dopamine. The obvious exceptions are the arthropods in which the chemically related biogenic amine octopamine has a greater effect on reward learning and reinforcement than dopamine. We review the functions of these biogenic amines in behavioral responses to rewards in different animal groups and discuss these findings in an evolutionary contex [3].

Tonse NK Raju [4]: "Sometimes, even the most obvious facts need to be reiterated. An infant suckling at his or her mother's breast is not simply receiving a meal, but is intensely engaged in a dynamic, bidirectional, biological dialogue. It is a process in which physical, biochemical, hormonal, and psychosocial exchange takes place, designed for the transfer of much needed nutrients, for building a lasting psychosocial bond between the mother and her infant. Among mammals, breastfeeding has evolved over millions of years as a multitiered interaction to meet the biological and psychosocial needs of the progeny, enhancing its well-being and survival chances, as well as complementing the nurturing role of the mother. this unique, dynamic process benefits both the mother and her infant. Breastfeeding needs to be considered quintessentially as a continuation of the more intense, intrauterine dialogue, mediated through the placenta and the umbilical cord between the mother and her fetus.

Whether feeding at the breast is complementary to the nutritional value of human milk, which might explain the diverse range of benefits to the mother and her infant, remains to be studied. innovative methods from different scientific disciplines, such as behavioral, cognitive, and developmental neurosciences, and social anthropology may be useful to study this unexplored territory. Among the many benefits from breastfeeding during the first year of an infant's life, the effects on long-term cognitive development and IQ have been most controversial. Some researcher contend that breastfeeding should be considered the social norm, and lower cognitive scores in infants fed formula should be considered abnormal. Sullivan asked, "Is it possible that some property in the infant formula may not be conducive to full cognitive development?" It is an interesting question that needs to be elucidated in future studies. Be that as it may, the above arguments require one to critically consider the cause-and-effect relationships between breast-feeding and cognitive outcomes. 2 recent publications answer the concerns about the causal relationships.

In a longitudinal study of neurodevelopmental evaluation Jedrychowski et al. assessed 468 infants of non-smoking women at $1,2,3,6$, and 7 years of age. Infants who were exclusively breastfed consistently demonstrated between 2.1 and 3.8 higher IQ points at each measurement session compared with those who received 
mixed feeding (human milk plus infant formula). The longer the duration of exclusivity of breastfeeding, the higher was the IQ benefit. In this and and similar studies,1-4 the overall IQ advantage from breastfeeding appear to be small, but the effect size is highly significant from a public health perspective. Improvements of even a few IQ points, especially at the lower end of the IQ distribution, will reduce the number of children who might otherwise need special education [4].

"We have seen in last 2 centuries different way to have a brain map using various strategies. Since from the BROADMAN theories we have seen the introducing of technologies to support this working method. (Old and new) as EEG, TC, PET, FMRI, MEG, NIRS ant other with the scope to differentiates brain area in order to show their specific activity. This has made possible to produce anatomic images and map about the different brain area to be related with some different functions / disfuncions. But what we can think is to create a new ANATOMIC brain map using the drugs and substances that show high Activity level in neurology field. a new pharmacology brain map can be obtained using different molecules or physiopathological conditions: BDZ GABA receptor, Barbiturate, Opioids, Neuroleptics, antiepileptic's, Anti depressive and ipnotics. Anti-migraine, Amphetamine, Anti Parkinson, Ant dementia, Antimuscarinics, Anticholinergic Analgesics, General anesthetics, Antistaminics, Poisons and toxins, Antipyretics, antihypertensive Addiction substanties, Ethanol, Nicotine, New smart drugs, Heavy metals. Vegetal substances, Cannabinoids, Oxygen and Co2, Toxic substances (as cyanide), insulin Food (involved in lepton metabolisms), carbohydrates level, metabolic toxic subst, MABS and many other drugs and substanties or phisiophatological conditions [5].

Howard D Weiss et al. [6]: Impulse control disorders and compulsive behaviors associated with dopaminergic therapies in Parkinson disease" and "Impulse control disorders (ICD) (most commonly pathologic gambling, hypersexuality, and uncontrollable spending) and compulsive behaviors can be triggered by dopaminergic therapies in Parkinson disease (PD) [6].

Swapnil Gupta et al. [7]: Drug dependence is a major cause of morbidity and loss of productivity. Various theories ranging from economic to psychological have been invoked in an attempt to explain this condition. With the advent of research at the cellular and subcellular levels, perspectives on the etiology of drug dependence have also changed. Perhaps the greatest advance has been in the identification of specific receptors for each of the drugs, their target neurotransmitter systems and the intracellular changes produced by them. These receptors also provide potential targets for treatment strategies of drug dependence. This study attempts to present the mechanisms in the development of dependence and the newer treatment strategies for the major drugs of abuse like alcohol, opioids, cannabis, nicotine cocaine. Human addictions are chronically relapsing disorders characterized by compulsive drug use, inability to limit the intake of drugs, the emergence of a withdrawal syndrome during cessation of the drug use. Dependence has been defined as a cluster of behavioral, cognitive and physiological phenomena that develop after repeated substance use. It includes a strong desire to take the drug, difficulties in controlling its use, persisting in its use despite harmful consequences, a higher priority given to drug use than to other activities and obligations, increased tolerance and sometimes a physical withdrawal state (International Classification of Diseases (ICD).

Drug addiction has been conceptualized as a complex and chronic disease process occurring in the brain, which is modulated by genetic, developmental and environmental factors. The most consistent and reproducible finding in drug addiction is that abused substances activate the mesolimbic dopamine system, which reinforces both pharmacological and natural rewards. The mesolimbic -system consists of dopaminergic neurons in the ventral tegmental area (VTA) and their axonal projections to terminal fields in the nucleus accumbens (NAc) and the prefrontal cortex. Opioids, alcohol, nicotine, cannabinoids and psychostimulants all act on this system to increase synaptic levels of dopamine (DA). All these substances have specific receptors in the brain and the increase in dopamine levels in the mesolimbic system is the final effect that they produce. Receptor-mediated activity is the principal mechanism by which any chemical messenger acts. Chemical messengers are regulatory macromolecules, usually proteins. Receptors have two major functions of recognition and transduction., each receptor has two domains, i.e., a ligand-binding and an effector domain. The ligand-binding domain has a hydrophilic and lipophilic region and is usually hetero-polymeric. The binding of the ligand causes a change in the quaternary structure of the receptor. Receptors have various effector mechanisms, which are broadly of four types:

a) G protein-coupled receptors (Gs, Gi, Gq and G13)

b) Receptors with intrinsic ion channels

c) Enzymatic receptors

d) Receptors regulating gene expression

One of the most dramatic advances in drug- abuse research has been the identification of the target of every major drug abuse substantia. This advance occurred with the advent of radioligandbinding techniques, the biochemical characterization of drug binding sites and ultimately, with the application of molecular biology to clone and isolate these structures. Drugs can upregulate or downregulate their receptors and their effector mechanisms. These changes are effected through genetic mechanisms and are implicated in the development of tolerance and withdrawal. Earlier biochemical data supported that the site of action of drugs was homogeneous. It is now known that there is great diversity in drug-receptor interactions. nicotine was thought to have a homogeneous class of binding sites in the brain. It is now known 
that there are many different oligomeric receptors that bind and are activated by nicotine. the diversity of the receptor types, the cross-modality of drug-receptor interactions are becoming more and more significant. It was earlier thought that drug use caused changes in the specific binding sites, inactivation mechanisms or levels of endogenous ligand. The diversity of drug receptors now forces a consideration of changes in the actual structure of the receptor molecule or changes in the distribution of these molecules on surface of the neuron. Drugs of abuse also have long-term effects resulting from the expression of genes activated as a consequence of the action of the drug." [7].

Nestler Ej [8]: Regulation of gene expression is considered a plausible mechanism of drug addiction, given the stability of behavioral abnormalities that define an addicted state. Among many transcription factors known to influence the addiction process phenomena, one of the best characterized is DeltaFosB, which is induced in the brain's reward regions by chronic exposure to virtually all drugs of abuse and mediates sensitized responses to drug exposure. Since DeltaFosB is a highly stable protein, it represents a mechanism by which drugs produce lasting changes in gene expression long after the cessation of drug use. Studies are underway to explore the detailed molecular mechanisms by which DeltaFosB regulates target genes and produces its behavioural effects. We are approaching this phenomenon using DNA expression arrays coupled with the analysis of chromatin remodeling--changes in the post-translational modifications of histones at drug-regulated gene promoters--to identify genes that are regulated by drugs of abuse via the induction of DeltaFosB and to gain insight into the detailed molecular mechanisms involved. Our research findings establish chromatin remodeling as an important regulatory mechanism underlying drug-induced behavioural plasticity, promise to reveal fundamentally new insight into how DeltaFosB contributes to addiction by regulating the expression of specific target genes in brain reward- pathways [8].

Kovács GL et al. [9]: Neuropeptides affect adaptive central nervous system CNS processes related to opiate ethanol and cocaine addiction. Oxytocin (OXT), a neurohypophyseal neuropeptide synthesized in the brain and released at the posterior pituitary, also is released in the central nervous system (CNS). OXT acts within the CNS and has been shown to inhibit the development of tolerance to morphine, and to attenuate various symptoms of morphine withdrawal in mice. In rats, IV self-administration of heroin was potently decreased by OXT treatment. In relation to cocaine abuse, OXT dose-dependently decreased cocaine-induced hyperlocomotion and stereotyped grooming behavior. Following a chronic cocaine treatment, the behavioral tolerance to the sniffinginducing effect of cocaine was markedly inhibited by OXT. Behavioral sensitization to cocaine, on the other hand, was facilitated by OXT. OXT receptors in the CNS--mainly those located in limbic and basal forebrain structures--are responsible for mediating various effects of OXT in the opiate- and cocaine-addicted organism. Dopaminergic
DA neurotransmission-primarily in basal forebrain structures--is another important biochemical mediator of the central nervous system effects of OXT. Tolerance to ethanol ( hypothermia-inducing effect of ethanol) also was inhibited by the OXT [9].

Tammy Saah [10]: Addiction from an evolutionary perspective, we may understand its underlying significance and evaluate its three-fold nature: biology, psychology, and social influences. In this investigation research it is important to delve into the co-evolution of mammalian brains and ancient psychotropic plants. Gaining an understanding of the implications of ancient psychotropic substance use in altering mammalian brains will assist in assessing the causes - effects of addiction in a modern-day context. By exploring addiction in this manner, we may move towards more effective treatment early prevention, treating the root of the issue rather than the symptoms.

As we find ourselves in the beginning of a new millennium periods, we are faced with challenges to our survival as a human population. Some of the greatest threats to our survival are sweeping epidemics that affect millions of individuals worldwide. Drug addiction, although often regarded as a personality disorder, may also be seen as a worldwide epidemic with evolutionary genetic, physiological, and environmental influences controlling this behavior. the use of drugs has reached all-time highs. On average, drug popularity differs from nation to nation. The United Nations Office on Drugs and Crime identified major problem drugs on each continent by analyzing treatment demand. From the 1998 to 2002, Asia, Europe, and Australia showed major problems with the opiate addiction, South America predominantly was affected by cocaine addiction, Africans were treated most often for the addiction to cannabis.

Only in North America was drug addiction distributed relatively evenly between the use of opiates, cannabis, cocaine, amphetamines, and the other narcotics. all types of drugs are consumed throughout each continent.

Among the different approaches for diagnosis, prevention, treatment of drug addiction, exploring the evolutionary basis of addiction would provide us with better understanding since evolution, personality, behavior and drug abuse are tightly interlinked. It is our duty as scientists to explore the evolutionary basis and origins of drug addiction so as to uncover the underlying causes rather than continuing to solely focus on the physiological signs and global activity of this epidemic phenomena. Too often the treatment of addiction simply works to alleviate the symptoms of addiction, dealing with overcoming the physiological dependence and working through withdrawal symptoms as the body readjusts to a non-dependent state of homeostasis. we must not only concentrate on this aspect of addiction when considering the global treatments and preventative programs. We must take into consideration that it is not purely the physiology of addiction we are battling. 
Drug addiction is thought of as an adjunctive behavior, or a subordinate behavior catalyzed by deeper, more significant psychological and biological stimuli. It is not just a pharmacological reaction to a chemical but a mode of compensation for a decrease in Darwinian fitness. There are three main components involved in substance addiction: developmental attachment, pharmacological mechanism, and social phylogeny including social inequality, dominance, and social dependence. Developmental attachment created by environmental influences, such as parental care or lack thereof, may influence children's vulnerability to drug addiction. Evolutionarily speaking, children that receive care that is more erratic may focus more so on short-term risks that may have proved to be an adaptive quality for survival in ancient environments. Compounding that attachment, the pharmacological mechanism describes the concept of biological adaptation of the mesolimbic dopamine system to endogenous substance intake.

These factors combined with the influence of social phylogeny create a position for predisposition to drug addiction. They attribute to the common belief that many substances of abuse have great powers to heal and is often the driving motivation for overuse and addiction. Evolutionary perspective shows an intermediate and fleeting expected gain associated with drug addiction correlated with the conservation in most mammals of archaic neural circuitry, most often being a falsified sense of increased fitness and viability related to the three components of drug abuse. The chemical changes associated with fitness and viability are perceived by mammals as emotions, driving human behavior. Human behavior is mediated primarily by dopaminergic and serotonergic systems, both of ancient origins probably evolving before the phylogenetic splits of vertebrates and invertebrates. 5-HT (serotonin), stimulated by a small range of drugs, mediates arousal. It is believed to be inhibited by hallucinogens and also helps control wanting for ethanol and cocaine consumption.

The cortico-mesolimbic dopaminergic system, on the other hand, is believed to be the target of a wide range of drugs, including marijuana and cocaine, increasing the transmission of dopamine to the nucleus accumbens. This system mediates emotion and controls reinforcement, and is the primary pathway acted on by antipsychotic drugs such as chlorprothixene and thioridazine. Problematic use of drugs develops into addiction as the brain becomes dependent on the chemical neural homeostatic circuitry altered by the drug. No matter the theory of drug addiction, there remains one constant: withdrawal is inevitable. As a drug is administered continuously and an individual becomes addicted, the brain becomes dependent on the presence of the drug. With an absence of the drug, withdrawal symptoms are experienced as the brain attempts to deal with the chemical changes. There are believed to be evolutionary origins of drug addiction, which will be discussed further, as well as a link between physiological addiction and the evolution of emotion [10].
Zimmerberg B et al. [11]: Some of the behavioral deficits caused by prenatal or postnatal alcohol exposure have been demonstrated to be ameliorated by environmental manipulations such as handling or environmental enrichment. This experiment, in contrast, investigated whether behavioral deficits due to prenatal alcohol exposure could be exacerbated by a stressful experience, early weaning. Pregnant dams were given either a liquid diet with $35 \%$ of the calories derived from alcohol, a liquid diet without alcohol to control for any effects of the liquid diet administration, or ad libitum food and water. Half of each litter were weaned at 15 days of age (early weaning) and half were weaned at 21 days of age (normally weaned). Offspring were weighed, tested for activity in an open field at 18 days of age, and trained to find a hidden platform in the Morris water maze at 22-24 days of age. Alcohol-exposed subjects who were weaned early were more impaired in spatial navigation ability than any other group. the combination of early weaning and prenatal alcohol exposure caused the slowest growth. All subjects exposed to alcohol, regardless of weaning condition, had greater latencies to find the platform than those from the two control groups. There was no synergistic effect of alcohol and stress on activity levels, but all early-weaned females were more active than normally weaned females; males did not show this effect. Thus, environmental stressors such as early weaning can compound detrimental symptoms of prenatal alcohol exposure. These results have implications for the understanding of the effects of the environment on neuronal plasticity [11].

Tim Clutton Brock [12]: Traditional interpretations of the evolution of animal societies have suggested that their structure is a consequence of attempts by individuals to maximize their inclusive fitness within constraints imposed by their social and physical environments. In contrast, some recent re-interpretations have argued that many aspects of social organization should be interpreted as group-level adaptations maintained by selection operating between groups or populations. I review our current understanding of the evolution of mammalian societies, focusing, in particular on the evolution of reproductive strategies in societies where one dominant female monopolizes reproduction in each group and her offspring are reared by other group members. studies of the life histories of females in these species show that dispersing females often have little chance of establishing new breeding groups and so are likely to maximize their inclusive fitness by helping related dominants to rear their offspring. As in eusocial insects, increasing group size can lead to a progressive divergence in the selection pressures operating on breeders and helpers and to increasing specialization in their behaviour and life histories. there is little need to invoke group-level adaptations in order to account for the behaviour of individuals or the structure of mammalian groups [12].

KD Broad et al. [13]: A wide variety of maternal, social and sexual bonding strategies have been described across mammalian 
species, including humans. Many of the neural and hormonal mechanisms that underpin the formation and maintenance of these bonds demonstrate a considerable degree of evolutionary conservation across a representative range of these species. there is also a considerable degree of diversity in both the way these mechanisms are activated and in the behavioural responses that result. In the majority of small-brained mammals (including rodents), the formation of a maternal or partner preference bond requires individual recognition by olfactory cues, activation of neural mechanisms concerned with social reward by these cues and gender-specific hormonal priming for behavioural output.

With the evolutionary increase of neocortex seen in monkeys and apes, there has been a corresponding increase in the complexity of social relationships and bonding strategies together with a significant redundancy in hormonal priming for motivated behaviour. Olfactory recognition and olfactory inputs to areas of the brain concerned with social reward are downregulated and recognition is based on integration of multimodal sensory cues requiring an expanded neocortex, particularly the association cortex. This emancipation from olfactory and hormonal determinants of bonding has been succeeded by the increased importance of social learning that is necessitated by living in a complex social world and, especially in humans, a world that is dominated by cultural inheritance [13].

Rainer Spanagel [14]: Face validity of animal models in addiction research. Humans and laboratory animals, such as monkeys, rats, and mice, voluntarily take drugs by different routes of administration, be it orally or intravenously. If unlimited voluntary intravenous access to heroin or cocaine is provided, laboratory animals can easily overdose to death. Lethal overdosing also frequently happens in drug users. Mice and rats can also voluntarily drink large quantities of alcohol, which leads to strong intoxication. These characteristic features seen in drug-taking behavior in laboratory animals resemble drug-taking behavior in humans and suggests a high degree of face validity. face validity is often a result of anthropomorphic interpretations of an animal's behavior. If, however, behavioral features are evolutionarily developed, real face validity is inferred. behavioral fear responses are critical to most species for survival and developed over millions of years. freezing behavior in response to a threatening stimulus, as seen in mice and humans alike, has real face validity. Since it is generally believed that psychoactive drug use in humans is a novel feature of our environment and cultural development, one wonders whether drug-taking behavior in laboratory animals actually resembles drug taking in humans?

Given the fact that the evolution of our human ancestors and of animals proceeded in a world rich in drugs, an alternative theory favors the idea that drug and alcohol intake by mammals and other species has always been an everyday occurrence. occasional and even long-term intake of psychoactive drugs produced by a variety of plants or alcohol ingestion through sugar-rich plant products susceptible to fermentation might be a behavior that has been shaped over millions of years. Interestingly, it was found that hominids adapted to metabolize alcohol long before humandirected fermentation. Using a paleogenetics approach, Carrigan et al resurrected digestive alcohol dehydrogenases (ADH4) from our primate ancestors to explore the history of primate-alcohol interactions and identified a single mutation occurring roughly 10 million years ago that endowed our ancestors with a markedly enhanced ability to metabolize ethanol.

This change occurred around the time that our ancestors adopted a terrestrial lifestyle. Because fruit collected from the forest floor contains higher concentrations of fermenting yeast and alcohol than similar fruits hanging from trees, this transition may also be the first time our ancestors were exposed to and adapted to substantial amounts of alcohol. These discoveries favor the idea that from an evolutionary perspective, alcohol and drug-intake behavior has been shaped over millions of years and should be considered a part of our normal behavioral repertoire. These evolutionary roots of alcohol- and drug-taking behavior in mammals support the real face validity of animal models of drug self-administration [14].

Adam T Shipley et al. [15]: The idea that addiction occurs when the brain is not able to differentiate whether specific reward circuits were triggered by adaptive natural rewards or falsely activated by addictive drugs exist in several models of drug addiction. The suitability of crayfish (Orconectes rusticus) for drug addiction research arises from developmental variation of growth, life span, reproduction, behavior and some quantitative traits, especially among isogenic mates reared in the same environment. This broad spectrum of traits makes it easier to analyze the effect of mammalian drugs of abuse in shaping behavioral phenotype. the broad behavioral repertoire allows the investigation of selfreinforcing circuitries involving appetitive and exploratory motor behavior, while the step-wise alteration of the phenotype by metamorphosis allows accurate longitudinal analysis of different behavioral states.

This paper reviews a series of recent experimental findings that evidence the suitability of crayfish as an invertebrate model system for the study of the drug addiction. Results from these studies reveal that unconditioned exposure to mammalian drugs of abuse produces a variety of stereotyped behaviors. Moreover, if presented in the context of novelty, drugs directly stimulate exploration and appetitive motor patterns along with molecular processes for drug conditioned reward. Findings from these studies indicate the existence of drug sensitive circuitry in crayfish that facilitates exploratory behavior and appetitive motor patterns via increased incentive salience of environmental stimuli or by increasing exploratory motor patterns. This work demonstrates the potential of crayfish as a model system for research into the neural mechanisms of addiction, by contributing an evolutionary, 
comparative context to our understanding of natural reward as an important life-sustaining process.

As individuals experience repeated exposure to opiates and other psychoactive drugs, vulnerable individuals enter an addictive cycle that is triggered by several mechanisms. These drugs initially function as reinforcers that strengthen behaviors associated with drug intake. After a short period of time, the resulting tolerance and dependence lead to progressively higher doses to maintain a desired effect. At this stage, compulsive drug-seeking behaviors become evident, even when paired with negative consequences. To identify useful targets for the development of future therapeutic interventions for drug-seeking behaviors, many studies explored the central components of drug-sensitive reward processes in both vertebrate and invertebrate species.

Much of these efforts have been focused on an evolutionary basis of drug reward as an entrenched process within natural reward- systems. Findings from most of these studies reveal that mammalian drugs of abuse typically exploit the natural reward systems, which align with the species' adaptive needs. These drugs function by supplanting the individual's inherent pursuit of its basic needs, such as nourishment, shelter, and reproduction, with a search for the drug instead. Findings from these research studies provided the opportunity to investigate common neural substrates underlying reward in a model system that has previously shown remarkable success under similar conditions and, to date, has provided major insights into wide-ranging behavioral occurrences. The importance of invertebrate model systems in drug addiction research is then highlighted. a series of experiments that support crayfish as a powerful invertebrate model system for the study of drug addiction are discussed.

\section{Neural Pathways in Drug Addiction}

Dopamine DA is considered the primary neural pathway underlying the neural causations of excitement, curiosity, and exploration. Several studies in the past have challenged a unitary role of the pathway in "pleasure." The common neural pathways surrounding mesolimbic dopaminergic neurons are commonly thought to mediate subjective reward and maintain reinforcement processes via hedonic affect. Dopamine alters behavior via incentive salience in which motivational components are applied to stimuli that have shown to be rewarding in the past. Mesolimbic and neostriatal dopamine systems exhibit residual reward capacity even after depletion of dopamine, which demonstrates a value in learning that is independent of hedonia and strict reward-based learning. The concept of "wanting" has been defined from the idea of reward-related stimuli conferring a motivational value to an organism, which is distinct from hedonia. The "wanting" mechanism may be modulated by dopamine systems via perceived attractiveness, rather than the traditional view of receiving pleasure, or "liking" a stimulus.
The distinction between "wanting" and "liking" is important as it appears that drug-mediated dopamine responses progress by "wanting" something more but "liking" it less; Drugs can be associated with certain contextual cues, such as a novel environment. In example, when an organism is conditioned to receive a psychoactive drug paired with a sensory cue, associated neural functions are activated in response to the environmental cue. In the absence of the drug itself, the effect goes so far to reactivate and sustain drug seeking behavior.

The dopaminergic pathways are responsible for feelings of desire and reward in humans through their influence on the ventral tegmental region, medial forebrain bundle and the nucleus accumbens, and can modulate compulsive behavior characteristic of drug addiction in several mammalian models. Dopamine is also implicated in a more direct learning process, in which mesolimbic dopamine neurons fire unconditionally in affiliation with natural rewards often associated with survival. this dopaminergic activity will shift from firing in response to the reward itself to firing in response to the cue that is predictive of the novel reward Although reward can be grouped into a few separate processes; an object's incentive value, the connective learning process of predictive cues and the object of attraction including the object's ability to produce hedonism are distinct in their own way and they each relate to a dopaminergic response that reinforces reward; It is seemingly paradoxical, that humans and animals are susceptible to addictive effects of cocaine, a neurotoxic chemical that has been shown to be evolutionarily adapted to protect the coca plant from insect herbivory by interfering with motor control in the organisms that consume coca plant. The dopaminergic system should be affected by cues that provide reward, not a plant neurotoxin that is designed to thwart predation. Many theories have been proposed that attempt to provide an evolutionary explanation for this phenomenon, ranging from co-evolution of herbivores and plants, to simple fundamental differences in response to the chemical by mammals compared to arthropods.

Theintroduction of invertebrate model in evolutionarily relevant studies of drug-induced reinforcement, compulsion, withdrawal, reinstatement, and addiction has greatly broadened this field of research. These systems have shown to be powerful tools in the understanding of the neuroanatomical and behavioral processes underlying the addictive process. Benefits of invertebrates, aside from being more cost effective, offering reduced moral concerns, and behaviors patterned by experimentally accessible neural structures, are shared homologies with mammals in the key neurochemical aspects of reward, including receptor elements, neuropharmacology, mechanisms of action, deactivation, and association with similar behavioral contexts. Monoamine systems developed during the transition to metazoan life, where they were used to adapt functions of individual cells to disturbances within their environment. dopamine and serotonin receptors predate the 
chordate lineage, and divergence has given rise to considerable diversity in specific subtypes within different lineages, along with some unique differences in receptor subunits and pharmacological properties in both vertebrates and invertebrates.

As a result of the divergence during evolution, mammals utilize oxidation and methylation while flies use $\mathrm{N}$-acetylation and $\beta$-alanylation for dopamine (DA) metabolism. Indeed, flies lack the genes required to synthesize norepinephrine and epinephrine, and these are two major catecholamines derived from DA that function in neuromodulation signaling in mammals. A cloned dopamine receptor from D. melanogaster has similar structural functional properties with vertebrate D1-type receptors, but the pharmacological properties are very different. The characterization of the sensitivity of D. melanogaster to cocaine in an in-situ hybridization study demonstrates that dopamine transporter (dDAT) lacks all the structural components that are found in the mammalian catecholamine transporters. Cocaine displayed a lower affinity for dDAT when compared with serotonin transporter. This study provides evidence that the structural and pharmacological profiles of dDAT is different from the DAT of vertebrate species. it indicates that injected cocaine, methamphetamine or morphine agonists or antagonists may function differently in vertebrate and invertebrate models of addiction. Despite the differences that exist between vertebrates and invertebrates, crayfish, D. melanonogaster and the other invertebrate model systems will continue to provide new insights into the regulatory mechanisms of DA signaling drug addiction research.

With the expansion of drug-addiction research into invertebrate models, identification of behavioral stereotypes and profiles have become evident. Fruit flies are a popular model system and have been shown to behaviorally sensitize in a fashion similar to that of the mammalian neurochemical and behavioral response to psychostimulants. Behavioral sensitization in fruit flies is regarded to have an opposite effect of tolerance and is characterized by an increased intensity of drug cravings and associated behaviors. an important commonality between the two models suggests that catecholamine circuits in flies bear a strong resemblance to the mammalian sensitization process for behavioral sensitization to occur in both flies and rats stimulation of the pre-synaptic monoamine sites must occur. The post-synaptic sites also play an important role in the cocaine response as flies that under-express these receptors exhibit a reduced response to an initial exposure to the drug. The opposite is true for mutants that over-express the receptor. In each of these mutant cases, the flies will not sensitize as the wild-type flies do. Vertebrate dopamine receptor antagonists can block cocaine-induced behaviors in fruit flies and planarians, strongly suggesting that dopamine is implicated in the resulting altered motor behaviors.

Tyramine has been revealed as a vital part of the sensitization process in a number of animals, including drosophila. Mutant individuals exhibiting lowered amounts of this amine are affected normally by the initial effects of cocaine but are less likely to sensitize. An increase in the individual's tyramine will result in a stereotypical sensitization akin to the wildtype counterparts. The per gene has an interactive role with tyramine, in that those lacking the gene will not undergo a normal sensitization process when stimulated with a vertebrate D2 agonist. The recent work revealing the activity of tyramine and the per gene in invertebrates has suggested that these processes could be conserved across a wide range of taxa. Tyramine has been likened to amphetamine's pharmacological profile as it inhibits membrane transporter uptake and alters synaptic catecholamines. This work on the transcription of the per gene has led to its demonstration in mammalian dorsal striatal regions receiving input from midbrain dopaminergic neurons A recent study identified genes for 34 distinct ion channel types, 17 biogenic amine and 5 GABA receptors, 28 major transmitter receptor subtypes including glutamate and acetylcholine receptors and 6 gap junction proteins-the innexins in the nervous system of Jonah crab (Cancer borealis) and the American lobster (Homarus Americans).

These genes are associated with neural function in the crustacean systems and could provide important new insights to understand the organization of circuits in the control of behaviors. recent studies indicate that an invertebrate system is a powerful tool that can be used to investigate the neuro-anatomical, molecular and behavioral processes underlying the addictive process. Highlighting these accomplishments is vital in showing how simpler model systems can lead to exploration and discovery in mammalian systems as well. The desire to more firmly establish invertebrate models in the study of drug addiction is driven by the lower cost, easy genetic manipulability of invertebrate models.

To prove their effectiveness as a model, the biological and behavioral overlap between the 2 separate model systems needs to be demonstrated. The invertebrate model has been well established in the rewarding properties for psychostimulants, opioids, alcohol, nicotine, caffeine. Analogous to the mammalian models, invertebrates also exhibit behavioral and motor stereotypes after the administration of cocaine. These research studies show that fruit flies and planarians exhibit increased locomotion and appetitive activities which strongly resemble corresponding behaviors in mammals. Fruit flies have also been shown to demonstrate functional tolerance via a central nervous system adaptation with the administration of ethanol, mimicking mammalian tolerance and behavioral adaptation.

Land snails learn to self-administer electric current pulses into areas of the brain associated with sexual behavior and not administer treatments for areas controlling escape. This suggests that land snails feature distinct pathways involved with reward and punishment. Planarians exhibit susceptibility to place conditioning, as individuals will switch to non-preferred environments if it is 
paired with a psycho-stimulant. This effect could be subsequently blocked by administering selective vertebrate D1, D2 antagonists. These research studies offer insight into potential mechanisms that remain unexplored within the crayfish model in drug addiction research. Crayfish as a model organism features a highly modular, experimentally accessible nervous system, yet capable of substantial behavioral complexity. With strongly conserved evolutionary mechanisms for behavioral sensitization, drug dependence, and drug-induced reward seeking, crayfish demonstrate significant vulnerability to human drugs of addiction. Research in crustaceans thus offers a valuable perspective for studying the neural implementation of conserved behavioral phenomena, including motivation, escape, aggression, drug-sensitive reward [15].

Tara M Mandalaywala et al. [16]: Weaning, characterized by maternal reduction of resources, is both psychologically and energetically stressful to mammalian offspring. Despite the importance of physiology in this process, previous studies have reported only indirect measures of weaning stress from infants, because of the difficulties of collecting physiological measures from free-ranging mammalian infants. Here we present some of the first data on the relationship between weaning and energetic and psychological stress in infant mammals. We collected data on 47 free-ranging rhesus macaque infants on Cayo Santiago, Puerto Rico, showing that faecal glucocorticoid metabolite (fGCM) concentrations were directly related to the frequency of maternal rejection, with fGCM concentrations increasing as rates of rejection increased.

Infants with higher fGCM concentrations also engaged in higher rates of mother following, mother following was associated with increased time on the nipple, suggesting that infants that experienced greater weaning-related stress increased their efforts to maintain proximity and contact with their mothers. Infants experiencing more frequent rejection uttered more distress vocalizations when being rejected; there was no relationship between rates of distress vocalizations and fGCM concentrations, suggesting a disassociation between behavioural and physiological stress responses to weaning. Elevated glu cocorticoid concentrations during weaning may function to mobilize energy reserves and prepare the infant for continued maternal rejection and shortage of energetic resources [16].

Karen E Adolph et al. [17]: This article reviews research on the development of motor behavior from a developmental systems perspective. We focused on infancy when basic action systems are acquired. Posture provides a stable base for locomotion, manual actions, facial actions. Experience facilitates improvements in motor behavior and infants accumulate immense amounts of experience with all of their basic action systems. At every point in development, perception guides motor behavior by providing feedback about the results of just prior movements and information about what to do next. Reciprocally, the development of motor behavior provides fodder for perception. motor development brings about new opportunities for acquiring knowledge about the world, and burgeoning motor skills can instigate cascades of developmental changes in perceptual, cognitive, and social domains.

Motor behavior includes every kind of movement from involuntary twitches to goal-directed actions, in every part of the body from head to toe, in every physical and social context from solitary play to group interactions. The development of motor behavior bridges the entire lifespan from the first fetal movement to the last dying breath. Although movements fundamentally depend on generating, controlling, and exploiting physical forces, managing forces requires more than muscles and biomechanics. At every point in development, adaptive control of movement relies on core psychological functions. Perception and cognition are required to plan and guide actions. Social and cultural factors spur and constrain motor behaviors. Motor behaviors, in turn, provide the raw material for perception, cognition, and social interaction. Movements generate perceptual information, provide the means for acquiring knowledge about the world, and make social interactions possible.

According to a developmental systems view, motor behaviors cannot be understood in isolation, divorced from the bodily, environmental, and social/cultural context in which they occur. Movements are inextricably nested in a body-environment system. The body and the environment develop in tandem. New or improved motor skills bring new parts of the environment into play and thereby provide new or enhanced opportunities for learning and doing. Caregiving practices facilitate and constrain motor development. As a consequence, differences in the way caregivers structure the environment and interact with their children affect the form of new skills, the ages when they first appear, and the shape of their developmental trajectory.

New motor behaviors can emerge from a mix of interacting factors, some so pervasive that we mistakenly take them for granted, and some so subtle or non-obvious that we fail to recognize the link. Developmental changes in one domain can have cascading effects on development in other domains, sometimes far afield from the original accomplishment. the context in which behavior develops can be very different for individual children, resulting in developmental pathways that sometimes converge at the same outcome and sometimes veer off in unique directions. This research article is organized around four basic action systems-posture, locomotion, manual actions, and movements in the face and head. We focus primarily on infancy period (when basic action systems are acquired) [17].

Clarac Fet al. [18]: In both vertebrates and invertebrates, the elaboration of locomotion, and its neural control by the central nervous system, are extremely flexible. This is due not only to the network properties of relevant sets of central neurons, but also 
to the active participation of mutually co-operative central and peripheral loops of neural projections and activity. In this chapter, we describe experiments in which the above concepts have been advanced by comparing locomotor properties in the adult vs. neonatal rat preparation. Data obtained from the in vivo vs. in vitro preparation, and swimming vs. walking behavior, suggest that the locomotor pattern progressively exhibited after birth corresponds to successive steps in the maturation of locomotor networks. Our work emphasises that during the late pre- and early postnatal period, three distinct neural entities--segmental sensory input, descending pathways, and motoneurons--play a key role in the maturation of locomotion and its neural control. We propose that the neonatal rat preparation is an excellent model for studying the conversion from immature to adult locomotion. Some neural controls are more clearly demonstrable in the developing animal preparation than in the adult because the latter exhibits an array of complex and redundant adaptive mechanisms [18].

Galbally $\mathbf{M}$ et al. [19]: Oxytocin is associated with the establishment and quality of maternal behavior in animal models. Parallel investigations in humans are now under way. This article reviews the current research examining the role of oxytocin in mother-infant relations, attachment, and bonding in humans. A systematic search was made of three electronic databases and other bibliographic sources for published research studies that examined oxytocin and mother-infant relations in humans, including attachment, maternal behavior, parenting, and motherinfant relations. Eight studies were identified, all of which were unique in their methodologies, populations studied, and measures used. Seven studies found significant and strong associations between levels or patterns of oxytocin and aspects of mother-infant relations or attachment.

Oxytocin appears to be of crucial importance for understanding mother-infant relationships. The findings of this review suggest that the pioneering, but preliminary, research undertaken to date is promising and that replication with larger samples is needed. Research that draws on more robust measures of attachment and bonding, as well as improved measures of oxytocin that include both central and peripheral levels, will elucidate the role of oxytocin in human mother-infant relationships. As the production of oxytocin is by no means restricted to mothers, the extension of the oxytocin studies to fathering, as well as to alloparental caregiving, would be an intriguing next step [19].

\section{Results}

Mammalians and their related instinct - behavior is a clear and an real evolutionary advantages. Various neurological systems are involved (circuits and neurotransmitters or hormone like dopamine, oxytocin, prolactin and also other related neuronal pathways). The rewards liking circuits, compulsive behavior, craving, wanting and some other neuronal circuits are deeply involved in addictions reported in biomedical literature.

\section{Conclusion}

In many addiction behavior is possible to verify that the subject involved present family or social problems. And a lack of this link (between single individual and his "caregiver") can produce this kind of disorder whit un psychological unbalance. Is possible to contextualize this fact with the vertebrate " mammalian "evolutionary pattern?. Mammalians are vertebrates that make dependent behavior a crucial aspect of their evolution and this present similarity in the pathways of addiction. Is possible to verify that some same neuronal circuits and related neuro-trasmitters or hormone are involved in physiology of breast-feed or reward mechanism in mammalian and also in addiction physio pathology. (dependency- addiction, reinforce, reward circuits compulsive behavior). But breast feed period of progeny is a limited time and determinate endogenous factors contribute in this Fact. (a sort of dependence is stopped): the weaning phases and related motor neuron maturation). This aspect must be adequately investigated to better understand also addiction pathology, drugs weaning mechanism and the pharmaco- toxicology implications. In some situation the border between physiology and pathology is very thin.

\section{References}

1. Luisetto M, Naseer A, Ghulam R M, Ahmed Y R, Farhan A K, et al. (2018) Endogenous Archeological Sciences: Anatomy, Physiology, Neuroscience, Biochemistry, Immunology, Pharmacology, Oncology, Genetics as Instrument for A New Field of Investigation? Modern Global Aspects for A New Discipline Open Access J Addict \& Psychol 1(3).

2. Marco Diana (2011) The Dopamine Hypothesis of Drug Addiction and Its Potential Therapeutic Value. Front Psychiatr 2: 64.

3. Andrew B Barron, Eirik Søvik, Jennifer L Cornish (2010) The Roles of Dopamine and Related Compounds in Reward-Seeking Behavior Across Animal Phyla. Front Behav Neurosci 4: 163.

4. Tonse NK Raju (2011) Breastfeeding Is a Dynamic Biological ProcessNot Simply a Meal at the Breast. Breastfeed Med 6(5): 257-259.

5. Luisetto M, Behzad Nili Ahmadabadi (2012) A New Physio-Anantomic Brain Map. scientia e ricerca current Opinions in Neurological Science Neurol Clin Pract 2(4): 267-274.

6. Howard D Weiss, Laura Marsh (2012) Impulse control disorders and compulsive behaviors associated with dopaminergic therapies in parkinson disease. Neurol Clin Pract 2(4): 267-274.

7. Gupta S, Kulhara P (2007) Cellular and molecular mechanisms of drug dependence: An overview and update. Indian J Psychiatry 49(2): 85-90.

8. Nestler EJ (2008) Review. Transcriptional mechanisms of addiction: role of DeltaFosB. Philos Trans R Soc Lond B Biol Sci 363(1507): 3245-3255.

9. Kovács GL, Sarnyai Z, Szabó G (1998) Oxytocin and addiction: a review. Psychoneuroendocrinology 23(8): 945-962.

10. Tammy Saah (2005) The evolutionary origins and significance of drug addiction. Harm Reduct J 2: 8 .

11.Zimmerberg B, Weston HE (2002) Postnatal stress of early weaning exacerbates behavioral outcome in prenatal alcohol-exposed juvenile rats. Pharmacol Biochem Behav 73(1): 45-52.

12. Tim Clutton Brock (2009) Structure and function in mammalian societies. Philos Trans R Soc Lond B Biol Sci 364(1533): 3229-3242.

13. KD Broad, JP Curley, EB Keverne (2006) Mother-infant bonding and the evolution of mammalian social relationships. Philos Trans R Soc Lond B Biol Sci (1476): 2199-2214. 
14. Rainer Spanagel (2017) Animal models of addiction. Dialogues Clin Neurosci 19(3): 247-258.

15. Adam T Shipley, Adebobola Imeh Nathaniel, Vasiliki B Orfanakos, Leah N Wormack, Robert Huber, et al. (2017) The Sensitivity of the Crayfish Reward System to Mammalian Drugs of Abuse. Front Physiol 8: 1007.

16. Tara M Mandalaywala, James P Higham, Michael Heistermann, Karen J Parker, Dario Maestripieria (2014) Physiological and behavioural responses to weaning conflict in free-ranging primate infants. Anim Behav 97: 241-247.
17. Karen E Adolph, John M Franchak (2017) The development of motor behavior. Wiley Interdiscip Rev Cogn Sci 8(1-2).

18. Clarac F, Brocard F, Vinay L (2004) The maturation of locomotor networks. Prog Brain Res 143: 57-66.

19. Galbally M, Lewis AJ, Ijzendoorn Mv, Permezel M (2011) The role of oxytocin in mother-infant relations: a systematic review of human studies. Harv Rev Psychiatry 19(1): 1-14. (c) This work is licensed under Creative

To Submit Your Article Click Here:

Submit Article

DOI: $10.32474 / 0 J N B D .2019 .02 .000140$

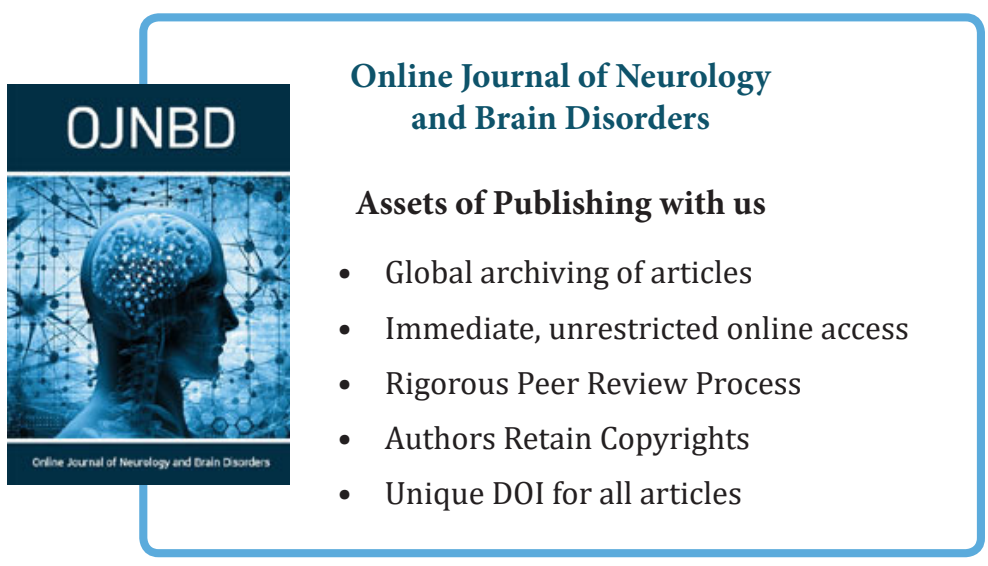

